\title{
LOCALLY GRADED GROUPS WITH ALL SUBGROUPS NORMAL-BY-FINITE
}

\author{
HOWARD SMITH and JAMES WIEGOLD
}

(Received 24 January 1994)

Communicated by R. B. Howlett

\begin{abstract}
In a paper published in this journal [1], J. T. Buckley, J. C. Lennox, B. H. Neumann and the authors considered the class of CF-groups, that is, groups $G$ such that $\mid H:$ Core $_{G}(H) \mid$ is finite for all subgroups $H$. It is shown that locally finite CF-groups are abelian-by-finite and BCF, that is, there is an integer $n$ such that $\left|H: \operatorname{Core}_{G}(H)\right| \leq n$ for all subgroups $H$. The present paper studies these properties in the class of locally graded groups, the main result being that locally graded BCF-groups are abelian-by-finite. Whether locally graded CF-groups are BCF remains an open question. In this direction, the following problem is posed. Does there exist a finitely generated infinite periodic residually finite group in which all subgroups are finite or of finite index? Such groups are locally graded and CF but not BCF.
\end{abstract}

1991 Mathematics subject classification (Amer. Math. Soc.): 20E26, 20 F50.

\section{Introduction}

A group $G$ is said to be a CF-group if every subgroup of $G$ has finite index over its core, that is, $H / H_{G}$ is finite for all subgroups $H$. If there is an integer $k$ such that $\left|H / H_{G}\right| \leq k$ for all $H$, then $G$ is said to be a BCF-group. It was proved in [1] that every locally finite CF-group is abelian-by-finite and BCF; this is a partial dual to a result of B. H. Neumann [3], which states that a group in which every subgroup has finite index in its normal closure is finite-by-abelian. However, the duality is imperfect. It was pointed out in [1] that the Tarski monsters of prime exponent $p$ are BCF and a long way from being abelian-by-finite. The aim of this article is to obtain a positive result along these lines, by imposing a relatively weak further hypothesis. A group is locally graded if every finitely generated nontrivial subgroup has a nontrivial finite image. We prove the following result.

(C) 1996 Australian Mathematical Society 0263-6115/95 \$A2.00+0.00 
THEOREM 1. Every locally graded BCF-group is abelian-by-finite.

We have been unable to decide whether every locally graded CF-group is abelianby-finite. Further discussion of this problem, which leads to some questions of independent interest, is postponed until Section 3. Our second result is easy to prove, and provides a hint as to what might go wrong in the general case.

THEOREM 2. Let $G$ be a $\mathrm{CF}-$ group such that every periodic image of $G$ is locally finite. Then $G$ is abelian-by-finite.

The class of groups satisfying the extra requirement of Theorem 2 is, of course, quite large. For instance, we see that every locally radical CF-group is abelian-byfinite. A result from [1] tells us that every finitely generated soluble CF-group is abelian-by-finite and BCF. Finite generation is important here. Let $A$ be the direct product of an infinite cyclic group and a $p$-quasicyclic group, and $G$ the splitting extension of $A$ by the automorphism of order 2 centralizing the cyclic group and inverting all elements of the quasicycle. It is easy to check the $G$ is CF but not BCF. Now $G$ is metabelian and of rank 3, and is even hypercentral when $p=2$, and so some obvious conjectures arising from [1] are disposed of. However, for nilpotent groups the situation is quite different.

THEOREM 3. Every nilpotent CF-group is BCF and abelian-by-finite.

\section{Proofs}

We begin with a couple of lemmas that reduce the proofs of Theorems 1 and 2 to a few lines.

LEMMA 1. Suppose that $G$ is a locally nilpotent CF-group. Then $G$ is abelian-byfinite.

PROOF. Let $N$ be the subgroup generated by all normal infinite cyclic subgroups of $G$. By Lemma 4.3 of [1], $N$ is abelian and its centralizer $C$ has index at most 2 in $G$. Since $G$ is locally nilpotent, this means that $C=G$ : no element of infinite order can be conjugate to its inverse. Let $A$ be a torsionfree subgroup of $N$ such that $N / A$ is periodic. Then $G / A$ is locally finite and hence abelian-by-finite, by [1]. Because of this, we may assume that $G / A$ is abelian and thus that $G^{\prime}$ is torsionfree. Let $T$ be the torsion subgroup of $G$. Then $G / T$ is locally nilpotent, torsionfree and abelian-by-periodic, and hence abelian. Thus $G^{\prime}$ is periodic and therefore trivial, so $G$ is abelian, and the proof is complete. 
LEMMA 2. Let $G$ be a locally graded periodic BCF-group. Then $G$ is locally finite.

PROOF. If the lemma is false, we may assume that $G$ is finitely generated and infinite. Let $H$ be the locally finite radical of $G$. By [1] and the CF-property, there exists a $G$-invariant abelian subgroup $K$ of finite index in $H$. By Lemmas 3 and 7 of [4], $G / H$ is then locally graded, and so we may assume that $H=1$. Since $G$ is BCF, there is a positive integer $k$ such that every subgroup of $G$ has index at most $k$ over its core. Thus, for every $g$ in $G,\left\langle g^{k !}\right\rangle \triangleleft G$. But $g$ has a finite order so $g^{k !}=1$, since the locally finite radical has been assumed trivial. Thus $G$ has exponent at most $k$ !.

At this point we depart from simplicity and use the fact that the restricted Burnside problem has a positive solution for all exponents; this is a consequence of the Classification Theorem and Zel'manov's celebrated solutions for prime-power exponents $[5,6]$. (Added in proof: We have now found a way of obviating reference to these deep results, so that there is a self-contained 'simple' proof.) What it means here is that there is a positive integer $n$ such that every finite image of $G$ has order at most $n$. But then the finite residual $R$ of $G$ has index at most $n$ in $G$, and is therefore finitely generated. Since $G$ is locally graded, it follows that $R=1$ and $G$ is finite. This contradiction completes the proof.

We turn now to the proof of Theorem 1. Let $N$ be as defined in the proof of Lemma 1 , and let $C$ be the centralizer of $N$ in $G$. By Lemma 1 of [4], $C / N$ is locally graded and hence, by Lemma 2 and [1], it is locally finite and abelian-by-finite. Thus $G$ is nilpotent-by-finite, and Lemma 1 applies to give the result.

The proof of Theorem 2 is very similar, and we omit it.

For Theorem 3, let $G$ be a nilpotent CF-group and $Z$ the centre of $G$. By Lemma 1, there is a normal abelian subgroup $A$ of finite index $n$, say, in $G$. Since $\left[A,{ }_{c} G\right]=1$ for some positive integer $c$, induction on $c$ gives that $\left[A^{n^{\prime}}, G\right]=1$, where $n^{\prime}=n^{c-1}$; it follows that $G / Z$ has exponent dividing $n^{c}$. We need to show that $G$ is BCF, and we begin by reducing to the case where $n$ is a prime-power. Suppose that $G=G_{1} G_{2}$, where $G_{1}$ and $G_{2}$ are normal subgroups of $G$ such that $A \leq G_{1} \cap G_{2}$, and that there exist integers $k_{1}$ and $k_{2}$ such that $\mid H /$ Core $_{G_{i}} H \mid \leq k_{i}, i=1,2$, for every subgroup $H$ of $A$. Let $L$ be an arbitrary subgroup of $A$, and write $K=$ Core $_{G_{1}} L$, so that $|L / K| \leq k_{1}$. Also, Core $K=\operatorname{Core}_{G_{2}} K$, so that this core has index at most $k_{2}$ in $K$. It follows that $L$ has index at most $k_{1} k_{2}$ over its $G$-core. This (together with an obvious induction) allows us to assume that $n=|G / A|=p^{k}$, for some prime-power $p^{k}$.

Let $B$ denote the $p^{\prime}$-component of $A$, suppose that there exists an integer $m$ such that every subgroup of $G / B$ is of index at most $m$ over its core, and let $H$ be any subgroup of $A$. Then there is a normal subgroup $K$ of $G$ such that $B \leq K \leq H B$ and $|H B / K| \leq m$. Now $K=B(K \cap H)$ and $K \cap H$ has index at most $m$ in $H$, so 
in order to show that $\left|H / H_{G}\right|$ is bounded, we may assume that $H B$ is normal in $G$. Take $x$ in $H^{G} \cap B$, so that $x=h \sigma$ for some $h \in H, \sigma \in[H, G] \leq[A, G]$. Then $\sigma^{n^{c}}=1$ and $x^{n^{c}}=h^{n^{c}} \in H \cap B$; since $B$ is a $p^{\prime}$-group we see that $x \in H \cap B$ and hence that $H^{G} \cap B \leq H$. Thus $H^{G}=H^{G} \cap H B=H\left(H^{G} \cap B\right)=H$, so that $H \triangleleft G$. Factoring by $B$, we may thus assume that the torsion subgroup $T$ or $A$ is a $p$-group. The argument splits into two cases depending on the finiteness or otherwise of the exponent of $T$.

If $T$ has finite exponent, then it is a direct factor of $A$ (see [2]), and we may write $A=T \times U$, where $U$ is torsionfree. Using the CF-property and arguing as in the proof of Lemma 2.1 of [1], we may assume that every subgroup of $T$ is normal in $G$. Let $H$ be any subgroup of $A$; then $H=(T \cap H) \times V$ for some torsionfree subgroup $V$ of $H$. If $U$ has finite rank, then $V$ also has finite rank $s$, say. By the CF-property, $V$ is then finite over a normal subgroup $W$, which is central since $[A, G]$ is periodic. Thus $V=\left\langle W, a_{1}, \ldots, a_{s}\right\rangle$ for suitable $a_{1}, \ldots, a_{s}$; since each $\left\langle a_{i}\right\rangle$ has index at most $n^{c}$ over its core and $T \cap H \triangleleft G$, this means that $H$ has bounded index over its core. Next, suppose that $T$ is finite. Then $G$ is centre-by-finite, since $U$ is finite over a central subgroup, and altogether we may assume that $T$ and $U$ are both of infinite rank. We shall prove once more that $G$ is centre-by-finite. If not, it is clear that there exists a countably infinite direct product $\left\langle a_{1}\right\rangle \times\left\langle a_{2}\right\rangle \times \cdots$ of cyclic subgroups of $T$ such that no $\left\langle a_{i}\right\rangle$ is central in $G$. Let $u_{1}, u_{2}, \ldots$ be free generators of a free abelian subgroup of $U \cap Z(G)$ of countably infinite rank, and set $h_{i}=u_{i} a_{i}$ for each $i$. Then $\left\langle h_{i}\right\rangle$ is not normal in $G$, since $h_{i}^{g}=u_{i} a_{i}^{g} \notin\left\langle u_{i} a_{i}\right\rangle$ since $\left[a_{i}, g\right] \neq 1$. However, $\left\langle h_{i}\right\rangle^{G} \leq\left\langle u_{i}, a_{i}\right\rangle$. Writing $S=\left\langle h_{1}, h_{2}, \ldots\right\rangle$, we see that $S$ does not have finite index over its core, a contradiction. Thus $G$ is centre-by-finite and therefore BCF. We may now assume that $T$ is of infinite exponent.

Let $B$ be a basic subgroup of $T$. If $B$ has finite exponent, then $A$ splits over $T$, so that $A=T \times U$ for some $U$, and $T=B \times D$, where $D$ is divisible [2]. Clearly, $D$ is central in $G$ and, as above, $B$ is a finite extension of a central subgroup and $G$ is yet again centre-by-finite. Thus we may assume that $B$ is of infinite exponent. As in the proof of Lemma 2.1 of [1], $B$ contains a subgroup $B_{1}$ of finite index all of whose subgroups are normal; arguing as in the proof of Lemma 3.8 of [1], we deduce that $B_{1}$ is central in $G$ and hence that $[B, G]$ is finite. For $a \in T, y \in G$ we have $a=a_{0}^{n^{c}} b$ for some $a_{0} \in T, b \in B$. Thus $[a, y]=[b, y]$ and $[T, G]=[B, G]$, so $[T, G]$ is finite. Our aim is to prove that $[A, G]$ is finite, but let us assume that this is so at this point. Then $G$ is finite-by-(centre-by-finite), and hence $G^{\prime}$ is finite, in particular $G$ is FC. But $G$ is in any case abelian-by-finite, so it is centre-by-finite and hence BCF.

Assume for a contradiction that $[A, G]$ is infinite. Since $[T, G]$ is finite, we may assume that $[T, G]=1$. Note that $[a, G]$ is finite for every $a$ in $A$, since $C_{G}(a) \geq A$ and $a$ has only finitely many conjugates and $[A, G]$ is periodic. There exists $a_{1}$ in $A$ such that $\left|\left[a_{1}, G\right]\right|=n_{1}>1$; setting $I_{1}=I_{A}\left(\left\langle a_{1}\right\rangle\right)$ for the isolator 
of $\left\langle a_{1}\right\rangle$ in $A$, we have that $A / I_{1}$ is torsionfree. Furthermore, $I_{1} T / T$ is torsionfree of rank 1 , so that, since $Z \geq T$ and $G / Z$ is of finite exponent, $I_{1} Z / Z$ is finite. Thus $\left[I_{1}, G\right]$ is finite since $I_{1}$ is finite $\bmod I_{1} \cap Z$ and $[a, G]$ is finite for every $a$ in $A$. The next step is to choose $a_{2}$ in $A / I_{1}$ such that $\left[a_{2}, G\right] \pm\left[I_{1}, G\right]$, and set $I_{2}=I_{A}\left(\left\langle a_{1}, a_{2}\right\rangle\right)$. Then $\left|\left[\left\langle a_{1}, a_{2}\right\rangle, G\right]\right|=n_{2}>n_{1}, A / I_{2}$ is torsionfree and $\left[I_{2}, G\right]$ is finite by arguments like those just used for $\left[I_{1}, G\right]$. Continuing in the obvious way, we find a subgroup $A_{0}=\left\langle a_{1}, a_{2}, \ldots\right\rangle$ such that $\left[A_{0}, G\right]$ is infinite and $A_{0} T / T$ is free. But then $A_{0} T=T \times V$, for some free abelian subgroup $V$, which, as before, contains a $G$-invariant and hence central subgroup of finite index. This gives the contradiction that $\left[A_{0} T, G\right]$ is finite, and the proof of Theorem 3 is complete.

\section{Concluding remarks}

As we observed in the introduction, we do not know whether every locally graded CF-group is abelian-by-finite. If $G$ is such a group and $N$ is defined as in the proof of Lemma 1 , then once again $G / N$ is locally graded. Let $H / N$ be the locally finite radical of $G / N$; then, by Theorem $2, H$ is abelian-by-finite. If $H \neq G$, then $G / H$ has no nontrivial normal locally finite subgroups, and hence the CF-property gives that all locally finite subgroups of $G / H$ are finite. As in the proof of Lemma 2, $G / H$ is locally graded.

These considerations result in the following observation. If there exists a locally graded CF-group which is not abelian-by-finite, then there exists a group $G$ of this sort that is finitely generated and periodic, in which every locally finite subgroup is finite. Clearly, the finite residual of $G$ must have infinite index; since factoring by a maximal normal abelian subgroup or a finite normal subgroup preserves residual finiteness, we may suppose that $G$ is residually finite and hence that every locally finite subgroup is finite. It is not clear whether we my factor by a normal subgroup $M$ which is maximal with respect to $G / M$ being infinite, since local gradedness could be lost. However, all of this indicates a special case that needs consideration, and we ask:

QUESTION 1. Let $G$ be a finitely generated, periodic, residually finite group in which every subgroup is either finite or of finite index in $G$. Is $G$ finite?

As a special case of this, we have:

QUESTION 2. Suppose that $G$ is a $p$-group satisfying the hypotheses of Question 1. Is $G$ finite?

Note that no infinite group $G$ satisfying the hypotheses of Question 1 can have an infinite abelian subgroup. It is not known, see [7, Problem 11.56], whether every 
finitely generated infinite residually finite group has an infinite abelian subgroup. Finally, one might hope to reduce the general question on locally graded CF-groups to one where the groups involve only finitely many primes, perhaps only one. We offer one last problem here.

QUESTION 3. Let $G$ be a finitely generated, residually finite, periodic CF-group in which every $p$-subgroup is finite, for all primes $p$. Is $G$ finite?

\section{References}

[1] J. T. Buckley, John C. Lennox, B. H. Neumann, Howard Smith and James Wiegold, 'Groups with all subgroups normal-by-finite', J. Austral. Math. Soc. (Ser. A) 59 (1995), 384-398.

[2] L. Fuchs, Infinite abelian groups (Academic Press, New York, 1970).

[3] B. H. Neumann, 'Groups with finite classes of conjugate subgroups', Math. Z. 63 (1955), 76-96.

[4] Howard Smith, 'On homomorphic images of locally graded groups', Rend. Sem. Mat. Padova, to appear.

[5] E. I. Zel'manov, 'Solution of the restricted Burnside problem for groups of odd exponent', Izv. Akad. Nauk. SSR Ser. Mat. 54 (1990), 42-59.

[6] _ ' 'Solution of the restricted Burnside problem for 2-groups', Mat. Sb. 182 (1991), 568-592.

[7] The Kourovka notebook (Unsolved problems in group theory) (Russian Academy of Sciences, Novosibirsk, 1992).

Department of Mathematics

Bucknell University

Lewisburg, PA 17837

USA

e-mail: howsmith@bucknell.edu
School of Mathematics University of Wales College of Cardiff Cardiff CF2 4AG

Wales e-mail: smajw@cardiff.ac.uk 